\title{
The (absence of a) relationship between thermodynamic and logical reversibility
}

\author{
O J E Maroney \\ Imperial College London \\ Physics Department \\ The Blackett Laboratory \\ Prince Consort Road \\ London \\ SW7 2BW
}

October 27, 2018

\begin{abstract}
Landauer erasure seems to provide a powerful link between thermodynamics and information processing (logical computation). The only logical operations that require a generation of heat are logically irreversible ones, with the minimum heat generation being $k T \ln 2$ per bit of information lost. Nevertheless, it will be shown logical reversibility neither implies, nor is implied by thermodynamic reversibility. By examining thermodynamically reversible operations which are logically irreversible, it is possible to show that information and entropy, while having the same form, are conceptually different.
\end{abstract}

\section{Introduction}

In a recent article on the information theoretic exorcism of Maxwell's demon, Bennett writes:

Landauer's principle, often regarded as the basic principle of the thermodynamics of information processing, holds that any logically irreversible manipulation of data ... must be accompanied by a corresponding entropy increase in non-information bearing degrees of freedom of the information processing apparatus or its environment. Conversely, it is generally accepted any logically reversible transformation of information can in principle be accomplished by an appropriate physical mechanism operating in a thermodynamically reversible fashion.(Bennett, 2003) 
In Bennett's, and in other papers (see Leff and Rex, 1990,2003; Zurek 1990) it appears that a strong claim is being made linking thermodynamic entropy to information processing in computers. In particular, it has been argued that the resolution of the Szilard Engine(Szilard, 1929) requires an understanding of principles of information processing.

In this paper it will be argued that, while Landauer erasure (Landauer, 1961) is essentially correct (contrary, for example, to the arguments of Earman and Norton $(1998,1999))$ it has been generalised incorrectly in its popularisation as "Landauer's Principle". When properly understood, it will be shown that information processing is unnecessary for resolving Maxwell's demon and that the strong connection between information and thermodynamic entropy is broken.

Two widespread generalisations of Landauer's paper, that may be concluded from Bennett's quote above, can be stated as:

- All logically irreversible operations require a generation of heat of $k T \ln 2$ energy per bit of information lost.

- While all logically reversible operations may be accomplished as a thermodynamically reversible process, some logically irreversible operations cannot.

We will be argue that neither generalisation is correct:

- Some logically irreversible operations can be implemented without generating heat.

- Any logically irreversible transformation of information can in principle be accomplished by an appropriate physical mechanism operating in a thermodynamically reversible fashion.

In Section 3 the implications of this for the thermodynamics of computation are considered, and then in Sections 4 and 5 this is applied to Shannon signalling and the Szilard Engine, respectively. The conclusion is that, while Shannon information and Gibbs entropy share the same measure, they are not equivalent, and that principles of information processing are not necessary to 'save' the second law of thermodynamics.

\section{Landauer Erasure}

Landauer Erasure $(L E)$ is a process by which a single information bearing degree of freedom, in one of two possible logical states, is restored to the logical value zero regardless of which state it initially occupies (Figure 1).

1. The logical state may be represented by an 'atom in a box', with the box divided in two by a moveable barrier. The atom on the left represents logical state zero and on the right, logical state one. The box is in contact with a heat bath at some temperature $T$. 


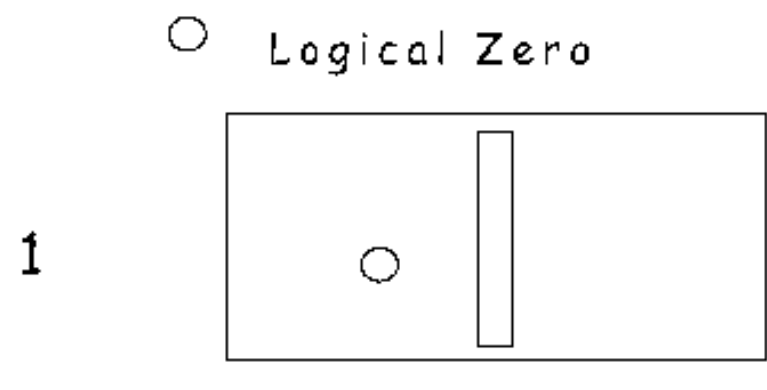

- Logical One
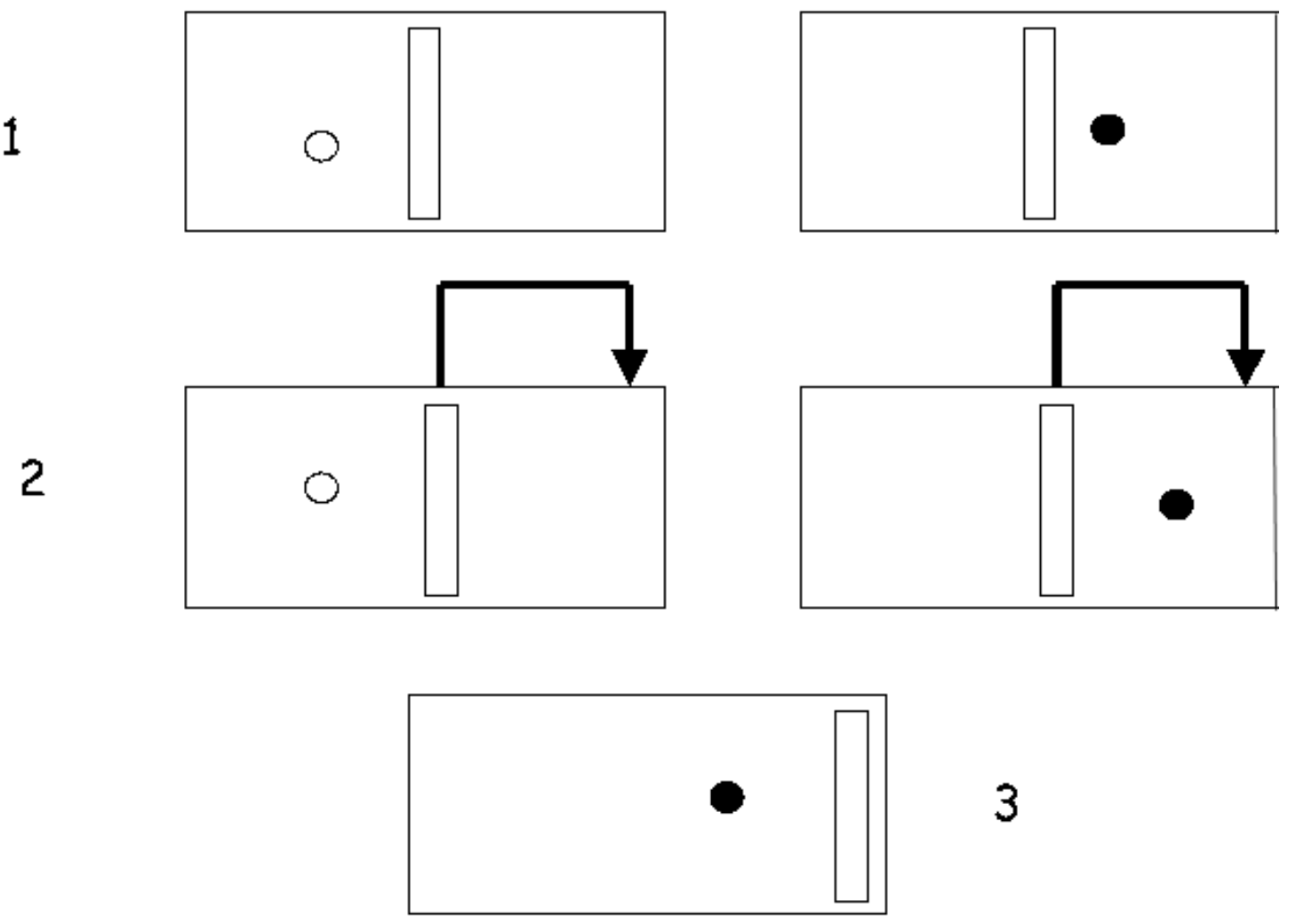

3

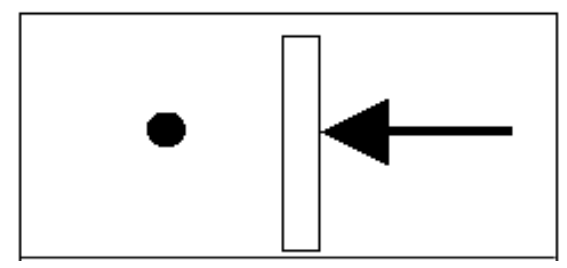

4

Figure 1: Landauer Erasure

2. The barrier is removed from the box and the atom is allowed to thermalise.

3. The barrier is inserted in the far right hand end of the box.

4. The barrier is isothermally moved to the centre of the box. This acts as the compression of an ideal gas so the work required is $N k T \ln 2$, where $N=1$. The work done on compressing the one atom gas is expelled into the heat bath $^{1}$.

There are two features of this process that should immediately be recognised:

\footnotetext{
${ }^{1}$ For justification that the ideal gas laws are valid for a single atom gas, see Leff (1995); Bender, Brody and Meister (2000) and Maroney (2002)
} 
- The compression stage (which requires the generation of heat) is not the same as the erasure stage (which actually destroys the information). It is noticeable that in their exposition of $L E$, Leff and Rex (1990 p.22-25) refer to "erasure/resetting". Stage 2 is the erasure, while Stage 4 is the resetting. A conclusion of our analysis will be that "erasure" must be understood as a separate operation to "resetting".

- The compression stage, Step 4 of the process, is an isothermal compression, and is thermodynamically reversible. The heat generation is not a thermodynamically irreversible process.

\subsection{Reversing Landauer Erasure}

Let us now consider the effect of reversing these Stages, in a "Reverse Landauer Erasure" (RLE).

1. The barrier is moved isothermally, reversibly to the far right of the box. Pressure from the expansion of the one atom gas can be used to extract $k T \ln 2$ energy from the heat bath and used as work.

2. The barrier is removed from the right hand end of the box.

3. After a thermalisation period, the barrier is inserted in the centre of the box.

4. The atom has an equal probability of being found on either side of the barrier.

It is apparent that if the logical states of the atom were initially uniformly distributed (ie. had probability $\frac{1}{2}$ of being on either side of the barrier) then the statistical state of the system is now exactly the same as it was before $L E$. The work used to reset the bit in Stage 4 of $L E$ is recovered in Stage 1 of $R L E$ and the entire process has taken place in a thermodynamically reversible manner.

While this point might be accepted by advocates of the information-entropy link, it is sometimes claimed that this is only true if the initial state of the system has uniform probability distribution:

memory erasure and resetting is always logically irreversible but it is thermodynamically reversible only when the initial memory ensemble is distributed uniformly among [0] and [1] states (Leff and Rex 1990)

If the initial statistical state of the bit is non-uniform, the RLE process will not leave the final statistical state in the initial distribution - the final state will still be probability half of being in either statistical state. As any non-uniform distribution will have lower entropy, the result is an uncompensated increase in Gibbs entropy and so is thermodynamically irreversible. 


\subsection{Non-uniform probabilities}

We will now show that the resetting of non-uniform probability distributions can also take place as a thermodynamically reversible process.

The key element here is to notice that the amount of information one wishes to reset is less for a non-uniform probability:

$$
H=-\sum p \log _{2} p
$$

This is 1 bit if the probabilities are equal, but less than 1 bit if the probabilities are non-uniform. As Landauer's principle states only that $k T \ln 2$ energy must be dissipated per bit of information, we should expect that with non-uniform probabilities the erasure and resetting may take place with a lower generation of heat.
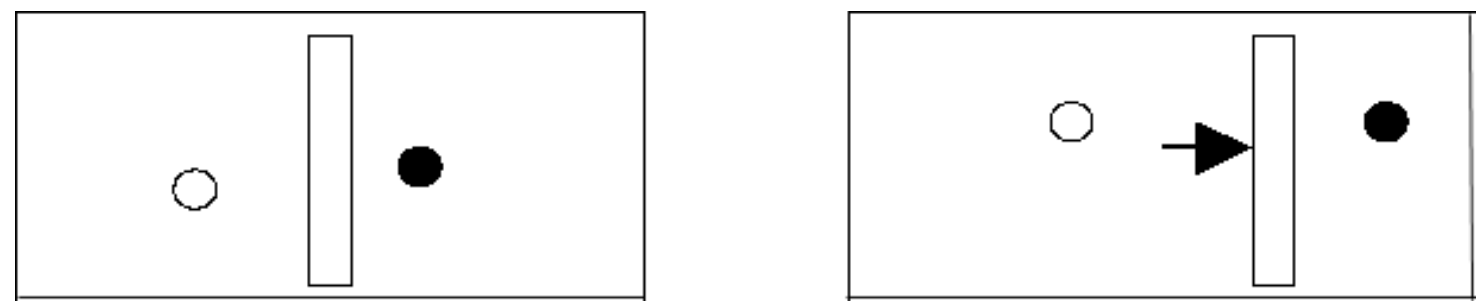

Figure 2: Landauer Erasure of Non-Uniform Probabilities

To achieve this lower generation it is necessary only to insert an additional Stage (Figure 2) between Stages 1 and 2 of the normal $L E$ :

- Isothermally, reversibly move the barrier to the position $x=p$ (where $x=0$ represents the barrier on the far left and $x=1$ the far right of the box).

If the atom is initially located to the left of the piston, with probability $p$, this requires a work expenditure of

$$
E_{L}=-k T \ln [2 p]
$$

while if it is initially to the right, the work expenditure is

$$
E_{R}=-k T \ln [2[1-p]]
$$

The mean expenditure of energy (over an ensemble of such operations) is

$$
E=p E_{L}+(1-p) E_{R}=(H-1) k T \ln 2
$$

When combined with the work in Stage 4 of $L E$, the total work required is

$$
W=H k T \ln 2
$$

in complete agreement with Landauer's principle ${ }^{2}$

Let us call this process $L E(p)$ to emphasise that the erasure and resetting operation is dependant upon the amount of information one wishes to erase.

If we now reverse $L E(p)$ we find that the operation $R L E(p)$ modifies $R L E$ as follows:

\footnotetext{
${ }^{2}$ See Shizume (1995), Piechocinska (2000) for proof that this is the least work required.
} 
- When the barrier is re-inserted (Stage 3) it is inserted at the location $x=p$ in the box. There is correspondingly the probability $p$ that the atom is found to the left, and $1-p$ that the atom is round to the right, of the barrier.

- The barrier is then isothermally, reversibly moved to the centre of the box, requiring a mean work expenditure of

$$
E^{\prime}=(1-H) k T \ln 2
$$

Now the final statistical state of the one-atom gas after $R L E(p)$ is identical to the statistical state before $L E(p)$. The net work required for $L E(p)$ exactly matches the net work extracted by $R L E(p)$ and the entire process has been achieved in a thermodynamically reversible manner.

It is important to note that the $L E(p)$ operation is not conditional upon the location of the atom. A measurement of, or correlation to, the logical state of the atom is not required. It is only tailored to the value of $p$, to the amount of information that is being reset. If the value of $p=0$ or $p=1$ the net work required for $L E(p)$ or $R L E(p)$ is zero. These correspond to the cases where the bit is known to be in a particular state, so the Shannon information is zero. In these cases, it is well known that operations exist ("Do nothing" and "NOT", for example) that can reset the bit to zero non-dissipatively.

The tailoring of the $L E(p)$ operation to the specific amount of information being reset makes it difficult to use this in practice. It is unlikely that an engineer designing a computer will be able to determine the statistical probabilities of the inputs to the logical operations the computer will perform. Simply assuming a uniform probability in all cases will not work. If, for example, the input to one operation was itself the output of an AND operation, then one or the other operation must have non-uniform probabilities for its input states.

Nevertheless, the key conclusion remains: in principle it is always possible to perform a logically irreversible operation in a thermodynamically reversible manner. Clearly we have not explicitly shown that all logically irreversible operations can be implemented as thermodynamically reversible processes, but provided that the thermodynamic process is tailored to the amount of information that is being manipulated, we see no reason that this cannot be done for any logically irreversible operation.

Perhaps the correct generalisation of Landauer's principle should be:

A logical operation needs to generate heat equal to at least ${ }^{3} k T \ln 2$ times the change in the total quantity of Shannon information over the operation.

Neither logical reversibility nor erasure of information appears in this formulation. Any operation may, in principle, be implemented as a physical process which reaches the limit and so can be made thermodynamically reversible.

\footnotetext{
${ }^{3}$ If the change in Shannon information is negative, then this gives the maximum amount of heat energy that can be extracted as work during the process.
} 


\begin{tabular}{|c|c|c|c|c|c|}
\hline \multicolumn{2}{|c|}{ LE } & \multicolumn{2}{|c|}{ RLE } & \multicolumn{2}{|c|}{ RAND } \\
\hline IN & OUT & IN & OUT & IN & OUT \\
\hline 0 & 0 & 0 & 0 & 0 & 0 \\
\hline 1 & 0 & 0 & 1 & 0 & 1 \\
\hline & & & & 1 & 0 \\
\hline & & & & 1 & 1 \\
\hline
\end{tabular}

Table 1: Logical Operations

\section{Logical Computation}

In the previous Section we considered the thermodynamic reversal of $L E$ and its generalisation to non-uniform probability distributions. We found that this could always be made thermodynamically reversible. We will now consider these as logical operations (for simplicity we will use $p=\frac{1}{2}$ from now on, although the conclusions can easily be generalised to the non-uniform case).

Logical reversibility is defined by Landauer (1961):

We shall call a device logically irreversible if the output of a device does not uniquely define the inputs.

It is clear from the truth tables (Table 10) that the operation $L E$ is logically irreversible. $R L E$, on the other hand, is logically reversible, if rather trivially so (given the output one can define what the input must have been - it must have been zero!)

If we now combine the two operations $(R L E)(L E)$ we get a new logical operation $R A N D$. This is also logically irreversible. The logical state of the bit after the computation cannot be used to deduce the logical state of the bit before the computation. Whatever information was originally represented by the bit has been lost. An 'erasure' of information has taken place (it must have taken place as Landauer Erasure is the first part of the process. Information that is 'erased' cannot be recovered!).

However, during ( $L E) k T \ln 2$ heat is generated, while during $(R L E) k T \ln 2$ is extracted from the environment. The final thermodynamic state is identical to the initial thermodynamic state ${ }^{4}$. So no net generation of heat has taken place. Nevertheless, a logically irreversible operation, involving the loss of 1 bit of information, has clearly taken place.

This directly contradicts the most common manner in which the generalisation of Landauer's principle is stated:

To erase a bit of information in an environment at temperature $T$ requires dissipation of energy $\geq k T \ln 2$. (Caves 1990, 1993)

in erasing one bit ... of information one dissipates, on average, at least $k_{B} T \ln (2)$ of energy into the environment. (Piechocinska, 2000)

\footnotetext{
${ }^{4}$ It is accepted by both Bennett (2003) and Leff and Rex (1990) that $L E\left(\frac{1}{2}\right)$ is thermodynamically reversible. We have here simply reversed the thermodynamic process.
} 
a logically irreversible operation must be implemented by a physically irreversible device, which dissipates heat into the environment (Bub, 2002)

We have shown that logically irreversible operations do not always imply a dissipation of energy into the environment.

In case it is argued that there is still some heat generation - during the $L E$ operation - but that we have just recovered this at a later stage, we should note that the combined operation $R A N D$ can be achieved without any work being required at any stage:

- Remove the barrier from the centre of the box.

- Wait for a time larger than the thermal relaxation time of the atom.

- Insert the barrier back into the centre of the box.

Now we have created exactly the same truth table as $(R L E)(L E)$ - so the original bit of information must again be considered lost or erased - and the statistical state remains the same at the end as at the beginning of the operation. The operation is logically irreversible, but is thermodynamically reversible and undergoes no heat exchange with the environment.

What we are seeing here is the consequences of one of the two facts we noted about Landauer Erasure above: the erasure stage of the operation is not the heat generation stage. It is the resetting operation (which compresses the probability distribution) which generates heat. The erasure stage, in which the logical irreversibility actually takes place and the original information is lost, does not require an exchange of energy with the environment.

\subsection{Non-deterministic operations}

The operations $R L E$ and $R A N D$ have unusual "truth tables". They certainly do not appear in most works on computational logic! The reason for this is that they display the converse property to logical irreversibility: logical non-determinism ${ }^{5}$. We will define this as follows:

We shall call a device logically non-deterministic if the input of a device does not uniquely define the outputs.

This should not be taken as requiring some kind of fundamental physical process that is non-deterministic (such as quantum wavefunction collapse) any more than logical irreversibility requires a fundamental physical process that is irreversible. In both cases an interaction with an uncorrelated environment (such as a heat bath) is all that is required.

\footnotetext{
${ }^{5}$ It is noticeable that in Bennett (2003), he refers throughout to deterministic computation.
} 


\subsection{Computational Paths}

Let us consider the computational paths that can be constructed from the addition of non-deterministic logical operations (Figure 3 ).

\subsubsection{Irreversible, Deterministic}

Type 1 computational paths are the most commonly encountered. In this we consider two possible input states (which for convenience we will assume are equiprobable) and at some point a merging of the computational paths takes place (such as by $L E)$. This requires a minimum amount of work to be performed on the system, which is expelled as heat.

\subsubsection{Reversible, Deterministic}

Type 2 paths are, in some respects, an improvement. Using reversible logic gates, such as the Fredkin-Toffoli gate, the output from the logical operation will contain additional bits which can be used to reconstruct the input states. Logical irreversibility is eliminated and no heat need be generated. All Type 1 computations may also be performed by Type 2 computational paths, as shown by Bennett (1973).

Type 1 and 2 computational paths are deterministic. If one restricts ones consideration to deterministic logical operations, then logical irreversibility implies heat generation. With the addition of non-deterministic logical operations, we find new computational paths are possible, whose thermodynamic properties are different.

Should we confine ourselves to deterministic logical operations? It seems unnecessarily restrictive to do so. Aside from the simple fact that such operations exist (and may even have computational value) there is the fact that the non-deterministic operation $R L E$ is just the thermodynamic reverse of $L E$. Leff and Rex (1990) and Bennett (2003) accept that $L E$ is thermodynamically reversible. For this to be meaningful, one must accept the reverse thermodynamic process as a legitimate operation. This reverse process is $R L E$ and is non-deterministic.

\subsubsection{Reversible, Non-deterministic}

Type 3 paths are logically reversible but non-deterministic. An example of such a process is the reverse Landauer Erasure, $R L E(p)$. It is interesting to note that this operation can extract heat from the environment and store it in a work reservoir. It cools a computer down, in contrast to Type 1 operations which heat computers up. Type 3 paths are generally the reverse of Type 1 paths (and vice versa).

This produces an interesting result. In Bennett (1973) a general procedure is devised for simulating Type 1 computations by Type 2 computations and so avoiding the generation of heat. This involves copying the output of the initial simulation ${ }^{6}$, then reversing all the logical steps, leaving only the input state and the copy of the desired output.

\footnotetext{
${ }^{6}$ This applies only to classical computation, see Maroney $(2001,2004)$.
} 
It can now be seen that there is an equivalent way of running a computer at a minimal thermodynamic cost, but without the restriction to using logically reversible computation. Perform the Type 1 computation, using irreversible logical operations and expelling heat into the environment. Then copy the output as before and perform the Type 3 computation that is the exact reverse of the original Type 1 computation. Now all the heat generated during the Type 1 computation can be extracted to perform work during the Type 3 computation!

This differs from the reversible simulation using Type 2 paths in two ways:

- For reversible simulation, the final state is the desired output and the original input state. With the non-deterministic reversal, the final state is the desired output plus a random choice of one of the possible input states that could produce the given output state, with a probability given by the statistical distribution of the input states.

- To achieve the minimal thermodynamic cost, it is necessary for the implementation of the separate irreversible and non-deterministic operations to be tailored to the statistical distribution of the input states. This would be very hard to achieve in practice.

Bennett's procedure for simulating Type 1 computations by Type 2 computations is therefore a far superior method of eliminating the conversion of work into heat than using a Type 3 computation to extract the generated heat back again.

\subsubsection{Irreversible, Non-deterministic}

Type 4 computational paths are irreversible and non-deterministic. Such computations may sometimes require a generation of heat, may sometimes be able to extract energy from the environment and may sometimes be implemented with no exchange of energy, depending upon the exact nature of the manipulation of the information. In the case of the $R A N D$ operation, no exchange of energy is required.

There is a curious asymmetry between irreversibility and non-determinism. Type 1 calculations can be performed by Type 2 operations. Type 3 and 4 operations, however, cannot be performed by Type 1 or 2 operations. This is because the nondeterministic operation can effectively operate as a random number generator ${ }^{7}$. A Type 3 or 4 operation can increase the algorithmic complexity of an input bit string. This is not possible for Type 1 or 2 operations.

This is especially curious when we consider that a Type 3 operation is just the reverse of a Type 1 operation, but if we reverse the simulation of a Type 1 operation by a Type 2 operation, we do not get a Type 3 operation!

All these computational paths can be accomplished in a thermodynamically reversible manner. However, one cannot tell directly from the truth table alone what

\footnotetext{
${ }^{7}$ Let it be clear the source of the random number is not necessarily through a non-determinism in the fundamental laws of physics. It is a computational non-determinism whose source is sampling the uncorrelated degrees of freedom of the environment.
} 
the reversible thermodynamic implementation will be - that requires the statistical distribution of the input states. The exception to this is Type 2 computations, which require neither generation nor extraction of energy, regardless of the statistical distribution.

\section{Shannon Information}

We will now briefly consider how the difference between Type 2 and Type 4 relates to the processing of Shannon information.

Start with the usual source and signal states occurring with probability $p$. Now suppose that this is arranged so that the energy of the states happens to be $E_{0}+$ $k T \ln p$ where $T$ is the temperature of the environment and $E_{0}$ is some constant. Now further suppose that the communication channel is very noisy. In fact over the course of the signal propagation, any individual state becomes completely thermalised.

The statistical state at the end of the transmission will be identical to the statistical state at the start of the transmission. However, one cannot say that the receiver is actually gaining any information about the signal transmitted. For information transmission, the individual state needs to be made stable against noise. Otherwise, it is not a signal at all.

For the thermodynamic state, there is no change. The thermodynamic entropy is the same at the start as at the end. Such thermalisation through contact with the environment is often an essential part of thermodynamic processes (such as isothermal compression and expansion of a gas). The individual state has no significance.

Of course, it remains the case that the receivers ignorance as to which state will be decoded remains $-\sum p \ln p$. What has changed is the significance, if any, the receiver may attach to the signal.

Consider a Type 2 computational paths and a Type 4 computational paths, where they differ only in the existence of a single $R A N D$ operation in the Type 4 path. Both before and after the $R A N D$ operation the statistical state of the Type 4 path is the same as the statistical state of the Type 2 path.

The quantity of Shannon information has not been changed by the $R A N D$ operation. This cannot be taken to mean that Type 2 and Type 4 paths are informationally equivalent! From the point of view of information processing, they are fundamentally very different types of operation. From the point of view of thermodynamics, however, the RAND operation has had no effect.

\section{The Szilard Engine}

Now let us turn to the Szilard Engine, and the use of Landauer's principle in the exorcism of Maxwell's demon (Figure 4). A single atom is contained within a box, with a moveable barrier (or piston) inserted in the centre of the box. There is a

probability $\frac{1}{2}$ of the atom being on either side of the barrier. The demon has a single memory register, which is initially in logical state 0 . 
The normal presentation of this, in terms of information, might go as follows:

1. The Demon performs a measurement upon the box to determine the location of the atom. The measurement generates no heat. The state of the Demon's memory now represents the location of the atom. As information has been gained about the location of the atom, its entropy has been reduced.

2. Having determined the location of the atom, the piston is moved to one side or the other, and the isothermal expansion used to lift a weight. This extracts $k T \ln 2$ energy, corresponding to the reduction in entropy of the atom during the measurement.

3. At first it seems that the 2 nd Law has been violated. However, there is still the information about the location of the atom recorded in the Demon's memory. To complete the cycle and return to its initial state, this record must be erased. According to Landauer's principle this requires the conversion of $k T \ln 2$ work into heat, exactly compensating for the work extracted.

Now let us consider how this is affected by the arguments of previous sections.

As we have seen, the Demon's one-bit memory can itself be physically realised by an atom in a box with a moveable barrier. Instead of resetting the Demon's memory, by an $L E$ operation, let us perform the $R A N D$ operation upon the Demon's memory, by pulling out and reinserting the barrier in the Demon's memory register. The result is a complete randomisation of the state of the Demon (Figure 5).

This operation is thermodynamically reversible and leaves the statistical state unchanged. Indeed, from the point of view of thermodynamics, nothing has happened. If there was a problem for thermodynamics, requiring resolution, before the $R A N D$ operation, that problem still exists after the $R A N D$ operation.

However, it causes problems for the information erasure story. There is no correlation between the state of the Demon's memory after the $R A N D$ and the state of the memory (or the initial location of the atom) before $R A N D$. The one-bit "memory" in the demon no longer represents the location of the atom. In fact it no longer represents information about anything. The $R A N D$ operation has already 'erased' the record of the Demon's measurement, it has 'erased' the information about the location of the atom, but has not generated any heat.

Is this a problem for thermodynamics? Hardly. The energy $k T \ln 2$ has been extracted, it is true. What is required, to 'save' the second law, is that there be a compensating increase of entropy of $k \ln 2$, and this has already happened. At the start of the process the Demon's 'memory' was in the logical state 0. At the end of the process it may be in either logical state with equal probability. Its entropy has increased by $k \ln 2$. This is precisely what is needed. As the Szilard Box itself is now back in its initial state, the entire process could simply be regarded as convoluted procedure for extracting $k T \ln 2$ energy from the isothermal expansion of the Demon's state, by an operation such as $R L E$ ! 
If it is not necessary to refer to Landauer Erasure after the $R A N D$ operation, then it was not necessary to refer to it beforehand. Why then has Landauer Erasure been promoted so strongly as the necessary principle to resolve the Szilard Engine? Historically, it was suggested by Brillouin (1951) and Gabor (1964) that the act of information acquisition (or performing a measurement) by the Demon must dissipate $k T \ln 2$ energy into the environment. It was Landauer's 1961 paper that demonstrated the falsity of this argument - they had wrongly generalised from certain measurement processes, which were dissipative, to assume all measurement processes must generate heat.

Having eliminated the generation of heat during the measurement process, it is natural to look for a different source of heat generation, and in the same paper Landauer appears to provide the answer: resetting is a heat generating operation, and requires the conversion of exactly the right amount of work into heat.

The essential irreversible act, which prevents the demon from violating the second law, is not the measurement itself but rather the subsequent restoration of the measuring apparatus to a standard state (Bennett, 1982)

information processing and acquisition have no intrinsic, irreducible thermodynamic cost, whereas the seemingly humble act of information destruction does have a cost, exactly sufficient to save the Second Law from the Demon. (Bennett, 2003)

This misses the point that it is not actually necessary to generate heat (or perform an irreversible act) to 'save the Second Law', it is only necessary to have a compensating entropy increase somewhere. The compensating increase in entropy has already taken place, and has taken place in the Demon itself.

If the increased entropy of the Demon is sufficient to save the second law after the $R A N D$ operation, when the memory register no longer represents information about the atom, then the fact that the memory register happens to represent information about the atom before the $R A N D$ operation is irrelevant to the resolution of the problem. The appeal to information acquisition, memory and erasure obscures the real reason why the second law is not violated. The movement of the barrier has to be correlated to the location of the atom. To do this requires an auxiliary system. This auxiliary ends up in a higher entropy state. This is not a principle of computation or of logic - it is a consequence of the physics of Hamiltonian dynamics. It is immaterial whether one considers the auxiliary to be a 'memory cell' or whether it can be regarded as representing 'information' about the state of the atom, or anything else.

\section{Conclusion}

Logically reversible operations may always be physically implemented as a thermodynamically reversible process. They may also, by being sub-optimally designed, be physically implemented as thermodynamically irreversible processes. 
We have shown the same holds true for logically irreversible operations. Any given quantity of information can be reset to zero in a thermodynamically reversible manner. For the limit of $k T \ln 2$ heat generation per bit to be reached, the thermodynamic process must be reversible.

In practice, logical operations are implemented by sub-optimal physical processes and so are thermodynamically irreversible. However, this irreversibility is not caused by the nature of the logical operation, it is by way of the operation being implemented by a thermodynamically sub-optimal physical process. This is as true for logically irreversible operations as it is for logically reversible operations.

This does not contradict Landauer (1961) in the least. All that Landauer can be said to have shown was that a resetting operation required a generation of heat in the environment. However, a confusion then appears to arise through the incorrect use of the term 'dissipation'. In Landauer (1961) and in much of the surrounding literature 'dissipation' is used more or less interchangeably with 'heat generation'. Strictly, dissipation should be used only when the conversion of work to heat arises through dissipative forces (such as those involving friction) which are thermodynamically irreversible. Forces which are thermodynamically reversible are non-dissipative ${ }^{8}$.

As an example, consider the isothermal compression of an $\mathrm{N}$-atom ideal gas to half it's volume. If this takes place sufficiently slowly then work equal to $N k T \ln 2$ must be performed upon the gas. The internal energy of the gas remains constant at $\frac{3 N}{2} k T$ throughout and the work done is converted to heat in the heat bath. All this heat generation is thermodynamically reversible as the free energy of the gas increases by $N k T \ln 2$ and the net change in entropy of the gas and heat bath is zero. In this case it would be incorrect to refer to the conversion of $N k T \ln 2$ work into heat as 'dissipation', as the force is frictionless, thermodynamically reversible, and so 'non-dissipative'.

In the case where $N=1$ we have exactly the isothermal compression of a oneatom gas that is used in Landauer Erasure. It should be clear, therefore, that when Landauer's minimum limit of $k T \ln 2$ heat generation is reached this is nondissipative. This can make statements such as

To erase a bit of information in an environment at temperature $T$ requires dissipation of energy $\geq k T \ln 2$ (Caves 1990,1993)

hard to understand when the equality is reached (and all the literature agrees that the equality can, in principle, be reached).

This confusion over the use of the term 'dissipation' seems to be the basis of Shenker's (2000) criticism of Landauer Erasure (which Shenker refers to as the 'Landauer Dissipation Thesis'), as Landauer Erasure can always be made 'nondissipative'. In contrast in this paper we have avoided the use of the term 'dissipation' where possible, and used instead 'heat generation'. Where 'dissipation' is used in the literature, we have assumed that 'heat generation' is all that was intended. Certainly Landauer (1961) can consistently be understood this way.

Nevertheless, the use of this term seems to have created a faulty link from 'heat generating' to 'thermodynamically irreversible':

\footnotetext{
${ }^{8}$ The author would like to thank John Hannay for discussions on this point.
} 
The existence of logically reversible automata suggests that physical computers might be made thermodynamically reversible, and hence capable of dissipating an arbitrarily small amount of energy (Bennett, 1973)

a logically irreversible operation must be implemented by a physically irreversible device, which dissipates heat into the environment (Bub, 2002)

We have clearly shown in this paper that logically irreversible devices are as capable of being made thermodynamically reversible (and hence non-dissipative) as logically reversible devices.

There is then a second confusion regarding whether logically irreversible operations must all be heat generating. What Landauer (1961) argues is that all unavoidably heat generating operations are logically irreversible. This is quite correct, but this does not mean that all logically irreversible operations are unavoidably heat generating. As we have seen, some logically irreversible operations need not generate heat. Some may even absorb heat and convert it to useful work!

We have seen that these operations remove any need to consider Landauer erasure or concepts of information processing, when resolving the 'problem' of the Szilard Engine. We would also argue that this demonstrates a conceptual difference between information and entropy.

The measure of the Shannon information clearly has the same mathematical form as the measure of the Gibbs entropy. Sharing the same mathematical form, however, does not make two things the same. An electrical circuit composed of a capacitor and an inductor can be described by the same mathematical equations as a mass on a spring, but this does not make mass the same thing as inductance.

The logically irreversible, non-deterministic $R A N D$ operation has a profound affect upon the informational state of a system, while leaving its thermodynamic state unaffected. The fact that the quantity of the Shannon information is the same before and after $R A N D$ should not lead us to think nothing has happened: Type 4 computational paths are profoundly different from Type 2 computational paths, as information processing systems. For thermodynamics, however, there is no difference. So there is more to a concept than its measure: information and entropy are not the same thing.

\section{References}

[1] C M Bender, D C Brody, and B K Meister. (2000) Quantum-mechanical Carnot engine. arXiv:quant-ph/0007002.

[2] C H Bennett. (1973) The logical reversibility of computation. IBM J Res Develop, 17:525-532.

[3] C H Bennett. (1982) The thermodynamics of computation - a review. Int $J$ Theor Phys, 21:905-940. Reprinted in Leff and Rex (1990). 
[4] C H Bennett. (2003) Notes on Landauer's principle, reversible computation, and Maxwell's demon. arXiv:physics/0210005.

[5] L Brillouin. (1951) Maxwell's demon cannot operate: Information and entropy I. J Appl Phys, 22:334-337. Reprinted in Leff and Rex (1990).

[6] J Bub. (2002) Maxwell's demon and the thermodynamics of computation. arXiv:quant-ph/0203017.

[7] C M Caves. (1990) Quantitative limits on the ability of a Maxwell demon to extract work from heat. Phys Rev Lett, 64(18):2111-2114.

[8] C M Caves. (1993) Information and entropy. Phys Rev E, 47(6):40104017.

[9] J Earman and J D Norton. (1998) Exorcist XIV: The Wrath of Maxwell's Demon. Part I: From Maxwell to Szilard. Stud Hist Phil Mod Phys, pages 435-471.

[10] J Earman and J D Norton. (1999) Exorcist XIV: The Wrath of Maxwell's Demon. Part II: From Szilard to Landauer and beyond. Stud Hist Phil Mod Phys, 30:1-40.

[11] D Gabor. (1964) Light and information. Progress in Optics, 1:111-153. Based on lectures delivered in 1951. Reprinted in Leff and Rex (1990).

[12] R Landauer. (1961) Irreversibility and heat generation in the computing process. IBM J Res Dev, 5:183-191. Reprinted in Leff and Rex (1990).

[13] H S Leff. (1995) Thermodynamic insights from a one-atom gas. Am J Phys, 63(10):895-905.

[14] H S Leff and A F Rex, editors. (1990) Maxwell's Demon. Entropy, Information, Computing. Adam Hilger. ISBN 0-7503-0057-4.

[15] H S Leff and A F Rex, editors. (2003) Maxwell's Demon 2: Entropy, classical and quantum information, computing. IoP. ISBN 0750307595.

[16] O J E Maroney. (2001) Sameness and oppositeness in quantum information. In Proceedings 21st ANPA Conference. ISBN 0952621568.

[17] O J E Maroney. (2002) Information and Entropy in Quantum Theory. PhD thesis, Birkbeck College, University of London. www.bbk.ac.uk/tpru/OwenMaroney/thesis/thesis.html.

[18] O J E Maroney. (2004) Are all reversible computations tidy? arXiv:quant-ph/0403079.

[19] B Piechocinska. (2000) Information erasure. Phys Rev A, 61:062314, 1-9. 
[20] O R Shenker. (2000) Logic and entropy. Philosophy of Science e-print archive. http://philsci-archive.pitt.edu/documents/dis0/00/00/01/15/index.html.

[21] K Shizume. (1995) Heat generation required by erasure. Phys Rev E, 52:3495-3499.

[22] L Szilard. (1929) On the decrease of entropy in a thermodynamic system by the intervention of intelligent beings. $Z$ Physik, 53:840. Reprinted in Leff and Rex (1990).

[23] W H Zurek, editor. (1990) Complexity and the Physics of Information. Addison Wesley. ISBN 0-201-51509-1. 
Type 1: Logically irreversible, deterministic

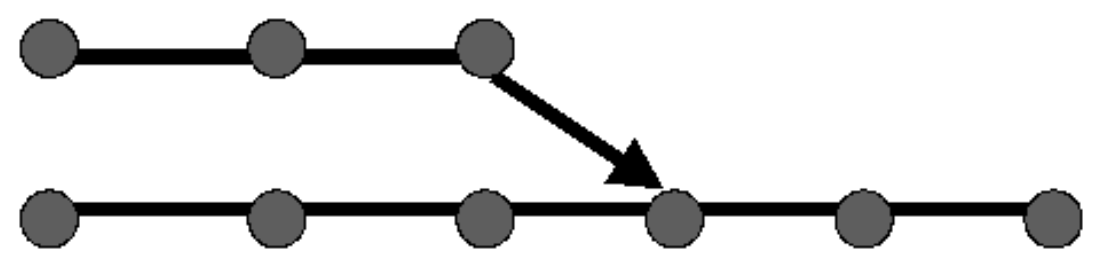

Type 2: Logically reversible, deterministic

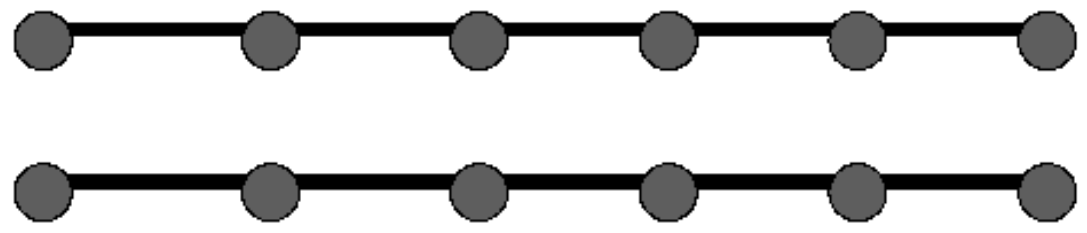

Type 3: Logically reversible, non-deterministic

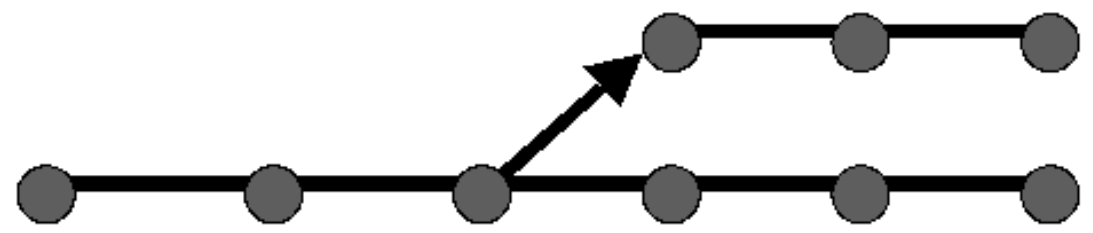

Type 4: Logically irreversible, non-deterministic

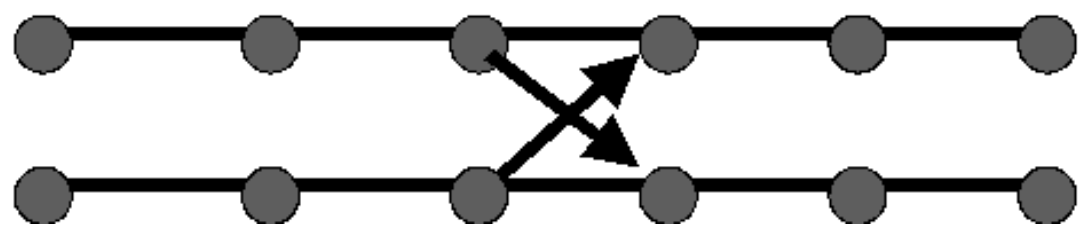

Figure 3: Computational Paths 

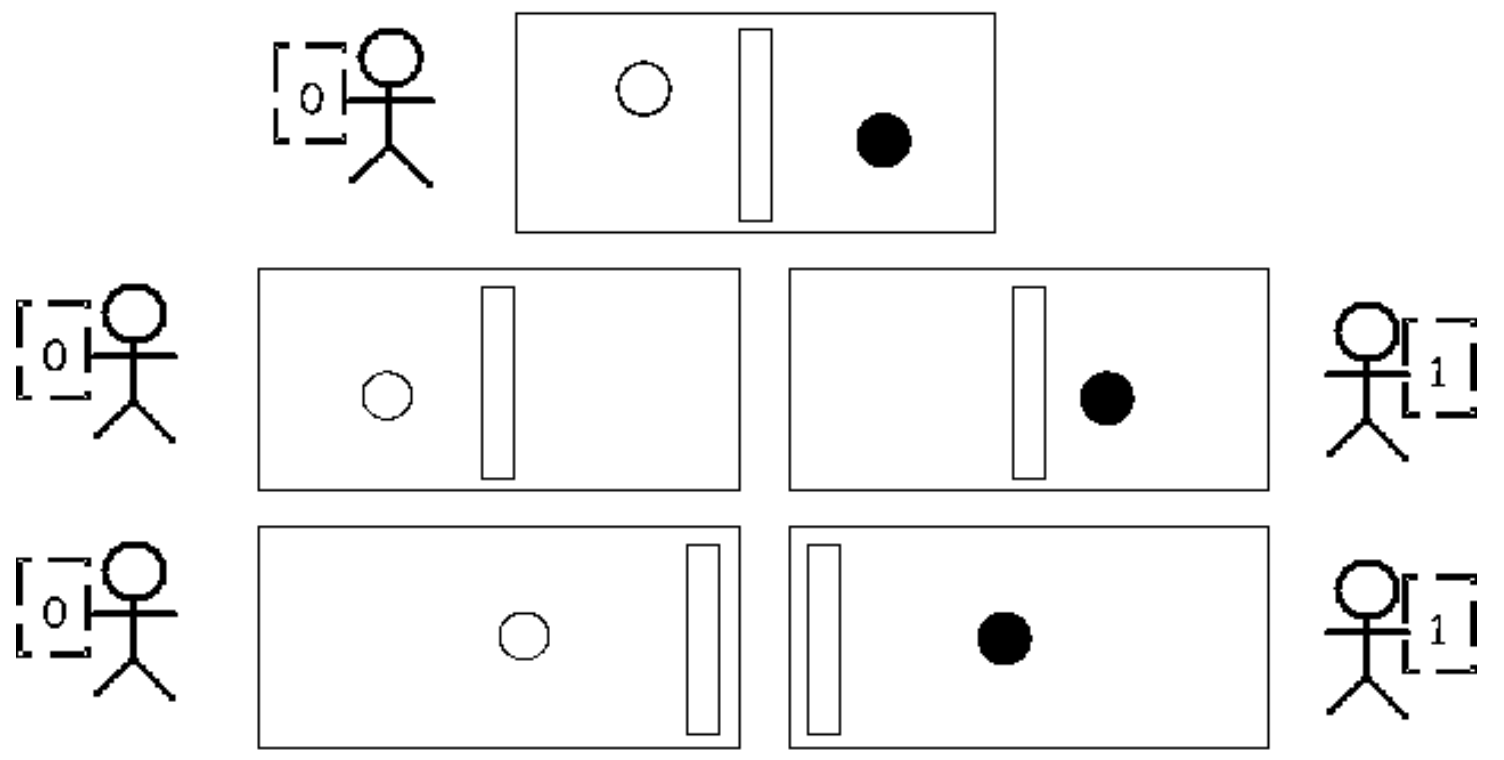

${ }_{01}$

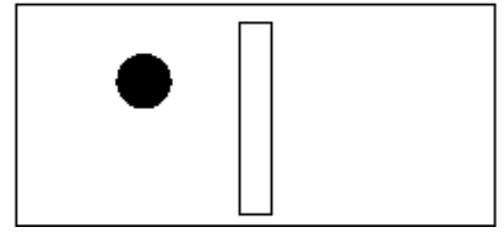

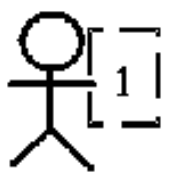

Figure 4: Landauer Erasure and the Szilard Engine
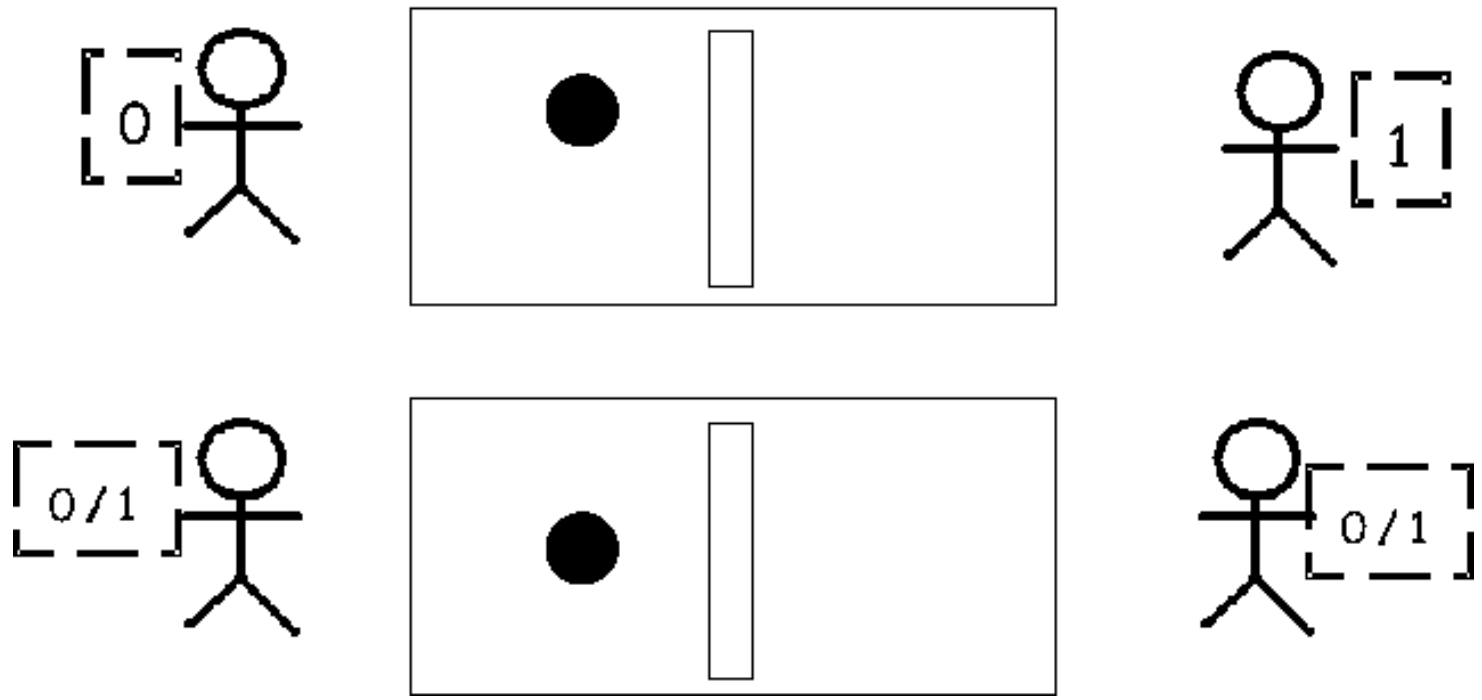

Figure 5: Randomisation of Demons "Memory" 